\title{
Los gobiernos locales y la cooperación transregional como alternativa a la política internacional de cambio climático
}

\author{
Claudia Ocman Azueta*
}

López Vallejo, Marcela. Reconfiguring Global Climate in North America. A Transregional Approach. Londres: Ashgate, 2014.

En los últimos años, los estudios sobre gobernanza global se han ocupado de diversos aspectos: desde definir el concepto intentando encontrar su fundamentación teórica, hasta crear nuevas herramientas metodológicas de análisis que permitan comprender mejor un objeto de estudio o, en su caso, contribuir a analizar la forma en que se llevan a cabo ciertos asuntos mundiales, lo que puede tanto inducir al análisis sobre cómo se conducen las políticas o las instituciones, o sobre cómo y a partir de qué se formulan las políticas y se establecen normatividades.

Si se compara, actualmente, el estado de la cuestión sobre gobernanza global en México, se puede decir que los estudios se han incrementado en un periodo de prácticamente diez años; sin embargo, aún no existe un solo criterio para definirla, ya que se trata de un concepto factible de ser abordado por varias disciplinas.

Por ejemplo, debido a la flexibilidad del concepto, existe la posibilidad de remitirse a distintos ámbitos de ubicación espacial y recaer en estudios de carácter global, regional o local. Fuera del ámbito espacial, existen otras opciones: se puede estudiar la totalidad de un tema, por ejemplo la economía mundial, o abarcar una parte de ésta, como las relaciones comerciales México-China. Fuera de estos aspectos y otros más que conciernen a las diferentes formas en las que se desarrolla un estudio sobre gobernanza global, existen puntos en común que recaen en la

\footnotetext{
* Profesora-investigadora en la Facultad de Derecho y Ciencias Sociales de la Benemérita Universidad Autónoma de Puebla (BUAP), <c_ocman@hotmail.com>.
} 
La gobernanza global es uno de los temas que forman parte de la agenda internacional, pues

a partir de las

cumbres internacionales fue incorporado a las políticas estatales. pluralidad de actores que participan en la gobernanza (uno de sus elementos característicos es la colaboración de otros actores, además del Estado, en los procesos de toma de decisión), así como en los vínculos que los actores establecen en el ámbito del diseño de los sistemas político-normativos para alcanzar un fin.

La amplitud de lo que en la práctica implica gobernanza y los distintos conceptos que sobre la misma existen, permiten abordarla con diferentes enfoques; así, se convierte en un marco analítico para delimitar un estudio, lo cual suele ser una opción que facilita analizar un tema dado, mediante el establecimiento de un estudio de caso.

Según lo que hemos planteado, la gobernanza global del medio ambiente o la gobernanza global ambiental (como se le denomina) se percibe como parte de la gobernanza global, ya que es uno de los temas que forman parte de la agenda internacional, pues a partir de las cumbres internacionales fue incorporado a las políticas estatales, y a pesar de que algunos consideran esfuerzos vanos en el sentido de que no se ha logrado un gran avance en la solución de los problemas ambientales, esta inclusión ha implicado el establecimiento de acuerdos internacionales, de políticas, de normas, así como la creación de instituciones, programas, etc., que fundamentan y dan forma a la gobernanza global ambiental.

Estudiar este tema resulta una tarea emprendedora que autores como Margaret Karns y Karen Mingst (2004) optaron por explicar, por ejemplo, a los actores que participan en la gobernanza global ambiental, quienes la conducen y también la problematizan. Otros especialistas intentan analizarla aunque, a pesar de que está parcializada por el tema ambiental, es complicado realizar este tipo de estudio, debido a la dinámica de la misma gobernanza, ya que los problemas ambientales son temas de magnitud no sólo en términos del impacto que se ha ocasionado a la naturaleza, sino en términos de los actores, de las políticas, etc., que ésta involucra, y también porque estos problemas son de muy diversa índole, por ejemplo, algunos son relativos al agua, otros a la pérdida de biodiversidad, otros a la desertificación, etcétera.

Encontramos, así, que la gobernanza global ambiental (que comparte los instrumentos jurídicos del derecho internacional ambiental, disciplina que contribuye a darle forma y delimitarla) está parcializada en subtemas o, diríase "subasuntos" que se intentan referir a las diferentes temáticas o problemáticas involucradas en un tema general como el del medio ambiente, para el cual se diseñan políticas que respondan a los 
compromisos internacionales, a objetivos particulares o grupales o que sigan determinadas tendencias.

La gobernanza global ambiental implica aspectos tan variados como regulaciones para el tránsito marítimo, políticas de manejo de las plantas nucleares y para el seguimiento de la sostenibilidad ambiental, medidas para la protección de los humedales o para la generación de energías alternativas; también involucra la participación de diversos actores de la sociedad civil, a empresas, instituciones internacionales, etc. Entre los problemas ambientales que ya son temas de estudio para varias disciplinas, invariablemente se encuentra el del cambio climático, cuyas repercusiones han llevado, en las cumbres internacionales, a la creación de instituciones como el Panel Intergubernamental en Cambio Climático, al involucramiento de científicos de las ciencias sociales y naturales, a la creación de redes entre actores públicos y privados, al establecimiento de políticas catalogadas como de largo y de lento alcance, de leyes en algunos casos (como el mexicano), entre otros.

Como asunto prioritario, el cambio climático despierta inquietudes e incertidumbres sobre el futuro de la humanidad, vinculadas a las condiciones ambientales que modificarán las condiciones de vida en el planeta; se ha convertido en un objeto de estudio que, desde las ciencias sociales, implicaría el análisis de las políticas y de las alternativas que en torno a su problemática se van estableciendo, de los actores involucrados, de los acuerdos suscritos y demás. Éste es el caso del libro Reconfiguring Global Climate in North America. A Transregional Approach.

Mediante un enfoque que parte de la construcción de un objeto de estudio creado a partir de las alternativas político-institucionales adoptadas en la región de América del Norte para dar seguimiento a la política internacional sobre cambio climático pero, sobre todo, a lo que la autora considera deficiencias de la forma en que se implementa esta política en lo global, se propone el concepto de regiones transnacionales de economías verdes (transnational green economic regions, TGER) como un espacio de gestión óptimo en el diseño de instrumentos de política en materia energética, esto con el fin de garantizar la provisión de bienes públicos, como el aire limpio, objetivo de la política de cambio climático.
Se propone el concepto de regiones transnacionales de economías verdes (transnational green economic regions, TGER) como un espacio de gestión óptimo en el diseño de instrumentos de política en materia energética. 


\section{CONSTRUCCIÓN DE UN ENFOQUE ANALítICO}

Inmersa en la complejidad teórica de la gobernanza global, López Vallejo construye su marco de referencia. Partiendo de diferentes definiciones y enfoques, analiza y resalta los elementos conceptuales y metodológicos útiles para su estudio, por lo que revisa las definiciones de varios conceptos de gobernanza global, clasificándolos con base en su criterio central para describir qué es esta gobernanza. En este sentido, indica cómo algunos conceptos concentran variables que implican a las redes sociales que se crean fuera del Estado, a las relaciones que se establecen por negocios internacionales, a las políticas ligadas al neoliberalismo las cua-

La institucionalización del medio ambiente mediante políticas

y normas fue un proceso inicialmente sectorializado a ciertos casos comúnmente asociados a la contaminación y a la protección de determinadas especies. les, como indica el texto, se expresan a través de la gobernanza corporativa y la mínima participación del Estado. Otros conceptos implican definiciones que ven esta gobernanza como una herramienta política, sinónimo de un proceso de formulación de políticas y de regulaciones en el cual existe una relación intrínseca entre estructuras y procesos en los que intervienen actores emergentes en diferentes contextos.

Consciente de las similitudes y de las diferencias teóricas y prácticas de la gobernanza, Marcela López propone un concepto ${ }^{1}$ que, basado en un enfoque radial (donde las partes conforman un todo), permite representar los nuevos esquemas de interacción que se producen entre actores a partir de un sistema de gobernanza específico; tal es el caso del medio ambiente y, en particular, del cambio climático. En este sentido, explica cómo se construyó la gobernanza global ambiental a partir de la protección parcializada del medio ambiente, común denominador de las políticas y de las normas que, como señalan otros textos (especialmente de derecho ambiental), como parte de su historia evolutiva, la institucionalización del medio ambiente mediante políticas y normas fue un proceso inicialmente sectorializado a ciertos casos comúnmente asociados a la contaminación derivada de las industrias y a la protección de determinadas especies de valor comercial, hasta que llegó un momento de reflexión: cuando se percibió al medio ambiente como un sistema integrado en el cual cada parte cumple una función. Esta perspectiva planteó la importancia de modificar las políticas y las normas, así como crear nuevas instituciones siguiendo un enfoque integral.

\footnotetext{
${ }^{1}$ Ella concibe la gobernanza como un proceso de coordinación entre diferentes actores y niveles de gobierno, que se presenta tanto en relaciones formales como informales en diferentes niveles, liderado por una autoridad (López Vallejo, 2014: 24).
} 
El parteaguas de los años ochenta, que correspondió a la suma paulatina de evidencias científicas y a las propuestas de nuevos modelos de desarrollo, condujo a que poco a poco la gobernanza global ambiental se integrara con una variedad de actores de índole pública y privada, así como por una amplia gama de instrumentos jurídicos internacionales, orientados a distintos temas, entre los que, como ya se dijo, suele destacar el cambio climático. En la gobernanza global ambiental, éste tiene su propio régimen, si bien vinculado a otros al considerar sus magnitudes e implicaciones, pero con los suficientes elementos integradores que permiten referirse a la denominación gobernanza global del cambio climático (la autora utiliza el concepto global climate governance).

\section{LOS PROBLEMAS DE LA GOBERNANZA GLOBAL DEL CAMBio Climático. Propuestas alternativas}

¿Cómo se construye la gobernanza global del cambio climático?, ¿cuáles son sus problemas?, ¿cuáles los retos y cuáles han sido sus obstáculos?, ¿cómo se implementa la política internacional sobre el cambio climático en los niveles regional y estatal?, ¿qué acciones se emprenden en América del Norte, cuáles son las metas, los obstáculos, pero, sobre todo, cuáles son las alternativas y sus alcances? Estos cuestionamientos básicos facilitan que comprendamos las aportaciones de este libro, en el marco de las preguntas de investigación establecidas (López Vallejo, 2014: 4) con las cuales se indaga sobre los problemas que enfrenta el Protocolo de Kioto, así como la gobernanza global del cambio climático ante la imposibilidad de cumplir con las metas de reducción de emisiones de gases de efecto invernadero (GEI) y, sobre todo, presentar alternativas que impliquen trasladar al ámbito de lo local (estatal o provincial en el caso de América del Norte), mediante políticas, los acuerdos internacionales, para proveer bienes públicos; se considera que éstos son la meta prioritaria de la gobernanza.

Con base en estas preguntas, se analizan las alternativas de la gobernanza global del cambio climático en un sentido crítico de la premisa "a problemas globales soluciones globales", que la autora confronta al plantear la importancia de encontrar actores con la capacidad de internalizar los problemas globales y que los conviertan en políticas, lo que implicaría relocalizar la autoridad para proveer bienes públicos globales como el aire limpio (López Vallejo, 2014: 5). En este sentido, se analizan
Se analizan las alternativas de la gobernanza global del cambio climático en un sentido crítico de la premisa "a problemas globales soluciones globales". 
los acuerdos institucionales para reterritorializar la provisión de bienes públicos globales, con una base transregional a través del mercado emergente de energéticos de carbono en América del Norte, buscando esclarecer cómo, desde lo regional, se brindan esos bienes públicos mediante otros diseños institucionales que abrirían alternativas que consideraran la relocalización de la autoridad, como ya mencionábamos, lo cual redefiniría el liderazgo de los actores involucrados en la gobernanza global del cambio climático; los gobiernos locales se convertirían en los actores principales de los procesos de regionalización.

\section{Desafíos en América del Norte PARA ENFRENTAR EL CAMBIO CLIMÁTICO}

Los gobiernos locales se convertirían en los actores principales de los procesos de regionalización.

Muchas veces las soluciones tienen que ser interiorizadas,

lo que amerita adoptar un enfoque que podría considerarse como de gobernanza multinivel.
¿Cómo trabajan los gobiernos locales en América del Norte y cuál es su finalidad? Partiendo de que la gobernanza global del cambio climático tiene deficiencias ${ }^{2}$ que derivan en dificultades para reducir los GEI, y que la finalidad de esta gobernanza es proveer bienes públicos globales, la autora trata de demostrar que los gobiernos locales son actores que en América del Norte implementan medidas para disminuir la contaminación por GEI, y que tales medidas contribuyen a proporcionar un bien público, como es el caso del aire limpio ya mencionado.

Acorde con esta lógica, si bien para mejorar la provisión de un bien público global se requieren soluciones globales, en los hechos se demuestra que los problemas globales no siempre se resuelven con soluciones globales, y que muchas veces las soluciones tienen que ser interiorizadas, lo que amerita adoptar un enfoque que podría considerarse como de gobernanza multinivel, propuesto por otros autores al referirse, sobre todo, al caso de la gobernanza en la Unión Europea.

En el caso de América del Norte, este tipo de gobernanza multinivel se reflejaría en las acciones regionales multinacionales que han tenido lugar mediante el Tratado de Libre Comercio de América del Norte (TLCAN) y, en especial, mediante el Acuerdo de Cooperación Ambiental y el Grupo de Trabajo en Energía; sin embargo, el único éxito evidente es que se influyera positivamente para proteger al medio ambiente. Debido a que el

\footnotetext{
${ }^{2}$ Respecto del Protocolo de Kioto, este texto se refiere a su diseño inadecuado, basado en enfoques nacionales, mientras los costos son regionales y por sector; a la carencia de métodos obligatorios de implementación, puesto que se establece la instrumentación voluntaria, así como a la no suscripción de países altamente contaminantes.
} 
cambio climático no es un tema prioritario como el comercio, existe una serie de restricciones que da como resultado un lento crecimiento en la cooperación ambiental regional. Esto, para la autora, demuestra que aunque el enfoque regional que se presenta en el TLCAN es correcto para emprender acciones más localizadas, indica que el formalismo regional en América del Norte ha llegado a su límite y, por tanto, enfrenta una alta dificultad para proveer bienes públicos; en otras palabras, se enfrentan dificultades para implementar medidas para reducir los GEI y proporcionar un bien público global como el aire limpio.

En este sentido, se debe abrir paso a otros conceptos en los que se preste atención a las acciones de los gobiernos locales (provinciales o estatales) como actores fundamentales en la política de cambio climático, ya que sus estrategias para reducir los GEI implican la creación de bloques regionales que inducen a acciones transnacionales basadas en la integración de políticas que se adaptan a las políticas nacionales e internacionales, a la vez que establecen relaciones con actores de diferentes niveles para implementar soluciones. En este contexto, surge -indica López Vallejo- un tipo de gobernanza transregional, con la finalidad de coproducir bienes colectivos globales, para lo que se requiere establecer vínculos con otras iniciativas del mismo carácter transregional.

\section{UN NUEVO MODELO DE REGIONALIZACIÓN}

¿Cuáles iniciativas reflejan un sistema de gobernanza transregional? En el caso del cambio climático y de la fundamentación teórica del texto, se propone a las ya mencionadas TGER, a las que la autora define como construcciones sociales con objetivos específicos que incluyen la integración y coherencia de actividades transnacionales. En éstas, la coherencia implica el diseño de sectores integrados, de políticas y estrategias para el clima, y acuerdos para el desarrollo sostenible (López Vallejo, 2014: 8).

En el sentido teórico que se propone en este libro para fundamentar el estudio de caso, las TGER son espacios que reinventan las fronteras y dan una nueva dimensión a la gobernanza global del cambio climático al relocalizar la autoridad y el liderazgo, lo que se interpreta como una especie de soberanía fragmentada en diferentes sectores de la política, y objetivos específicos en los que es necesaria la cooperación entre distintos niveles de autoridad y actores para reducir los GEI (López Vallejo, 2014: 8).
Existe una serie de restricciones que da como resultado un lento crecimiento en la cooperación ambiental regional. 
El estudio de caso conduce al lector a dos ejemplos de tGer que en América del Norte se construyeron en torno al sector energético, que es el sector con más emisiones de GEI.
El estudio de caso conduce al lector a dos ejemplos de TGER que en América del Norte se construyeron en torno al sector energético que, al igual que en el resto del mundo, es el sector con más emisiones de GEI. Estas TGER son la Iniciativa Regional del Gas de Efecto Invernadero (Regional Greenhouse Gas Initiative, RGGI) y la Iniciativa Climática de Occidente (Western Climate Initiative, WCI). Se analizan ambas TGER comparando las estrategias de los gobiernos locales con las relaciones que establecen con los gobiernos nacionales, en cuanto a su ámbito de jurisdicción y formulación de políticas sobre cambio climático y energía, con la intención de explicar el tipo y nivel de integración, ello con el fin de demostrar qué tan efectiva ha sido la relocalización de autoridad en términos de las oportunidades que proporcionan un enfoque transregional del cambio climático en América del Norte.

\section{LAS REGIONES TRANSNACIONALES DE ECONOMÍAS VERDES: ENTRE SUS LOGROS Y ALGUNOS OBSTÁCULOS}

Después de la justificación teórica que permite la posibilidad de que las TGER se consideren unidades de análisis y modelos de gestión útiles en la implementación de medidas de cooperación referentes a la política internacional del cambio climático, López Vallejo se aboca a sus componentes, forma de operación y alcances, con la intención de explicar cómo se presenta la gobernanza del cambio climático en un ámbito transregional. Mediante una serie de argumentos, explica los avances que estas regiones han tenido, pero también señala cuáles son sus dificultades.

Para ello presenta la estructura de las TGER y resalta el papel de los gobiernos locales como actores principales, cuyas acciones han logrado que sean reconocidos como tales (aunque de índole no gubernamental) en los foros internacionales; su presencia ha dejado en claro que existe una desconexión entre la política global y los problemas locales. Que estos gobiernos funjan como actores centrales permite que el diseño de políticas se base con mayor proximidad en los problemas, ya que se parte del hecho de que estos gobiernos son las instancias más cercanas a la ciudadanía y, por ende, de representación pública.

Por otra parte, se subraya cómo el establecimiento de relaciones transfronterizas antes y después del TLCAN ha permitido a realizar cierto tipo de actividades en cada región, dando como resultado la complementación 
de sectores económicos como el industrial, el comercial y el energético; en consecuencia, se han establecido intereses comunes alrededor de metas compartidas, para lo que se consideran las diferencias y el nivel de integración de las economías estatales. Tal es el caso del sector energético, en torno al cual se han implementado medidas que dan seguimiento a la política global de cambio climático mediante la instrumentación, por ejemplo, de políticas energéticas que permiten compartir costos y establecer un mercado de combustibles de carbono.

Precisamente en torno al mercado de carbono es donde surgen varias de las críticas a las TGER: algunas aluden a las interferencias al comercio ocasionadas por los requisitos impuestos a las industrias del carbono que operan fuera de las TGER; otras, se refieren a la superposición que se presenta entre las disposiciones nacionales y las que se establecen en las TGER, por ejemplo, en cuanto a sanciones que, en el caso de Estados Unidos, son competencia de la Agencia de Protección Ambiental.

Algunas otras críticas se inscriben en las inconsistencias entre la actuación de los gobiernos locales y nacionales (como el caso de Estados Unidos, que no aceptó el Protocolo de Kioto), provocando que los gobiernos locales de las TGER suscriban acuerdos con países extranjeros sin requerir la autorización del Congreso estadunidense, con lo que se violentan disposiciones constitucionales (López Vallejo, 2014: 176-179).

Por otra parte, existen reticencias (señaladas en el texto) que cuestionan el carácter transnacional como un indicador para delimitar una TGER. Para la autora, esto no presenta un obstáculo muy fuerte, ya que, si bien mientras la RGGI no tiene un carácter transnacional, porque sólo está integrada por estados de Estados Unidos y algunas provincias de Canadá como observadoras, la wCI sí es transnacional, debido a que abarca la costa oeste, desde Alaska hasta California, además de que incluye como observadores a algunos estados mexicanos. Acorde con la lógica del libro, el caso de la RGGI se toma como un reto que no devalúa los logros de las TGER.

Como proceso para la integración de un mercado de carbono, en el contexto de la RGGI y de la WCI, las TGER son casos loables; por ejemplo, a partir de su creación han fomentado la innovación, constituyen un modelo basado en iniciativas transregionales, difundible para construir otros mercados de carbono en una escala menor, lo que daría mejores resultados que un mercado de carbono global, sobre todo si se refuerzan los marcos institucionales y se reevalúa la presencia de los gobiernos locales en los foros internacionales y en la gobernanza global del cambio climático. 
En general, se espera que con la fijación de metas de reducción y con la homologación de los límites máximos

y de comercio se establezca un mercado de carbono que contribuya a la reducción de los GEl.

La flexibilidad presente en el caso de la RGGI, donde se permite a sus miembros vincularse con diferentes niveles de gobierno regional, nacional o global, es otra garantía de la capacidad de las TGER de actuar en diferentes espacios no restringidos al ámbito regional o transregional. Entre otros aspectos, este texto induce a considerar que tanto la RGGI como la WCI pasan por un proceso de integración que, como tal, va estableciéndose, y en su camino han logrado reducir los GEI. En el caso de la RGGI, se ha logrado descarbonizar más rápidamente su economía, en comparación con la wcI, al utilizar fuentes energéticas que no son de carbono, como la energía nuclear y el gas natural. En lo que corresponde a la wCI, ha de valorarse su capacidad de liderazgo al impulsar los instrumentos y las políticas en la región. En general, se espera que con la fijación de metas de reducción y con la homologación de los límites máximos y de comercio se establezca un mercado de carbono que contribuya a la reducción de los GEI.

\section{REFLEXIÓN FINAL}

Ante lo antes expuesto, se concluye que lo interesante del texto es la tentativa de una aportación distinta al concepto de gobernanza global mediante el análisis de la dinámica regional, por estados y provincias en los tres países de América del Norte; plantea, incluso, una alternativa al modelo de gobernanza global y a los modelos de gobernanza nacional ${ }^{3}$ aplicados al cambio climático que, precisamente debido a su poco alcance, abren paso a la actuación de actores estatales o provinciales, los que, por afinidades o complementariedades de sus sectores productivos, implementan medidas que ayudan a controlar el cambio climático, en seguimiento de la política internacional.

Si bien, como señala la autora, aún existen varios retos para las TGER, estas regiones serían una alternativa a las fallas de los mecanismos de mercado establecidos en el marco del Procotolo de Kioto. Como indica López Vallejo, la RGGI y la wCI son los dos mercados de carbono más importantes en América del Norte; aquí se manejan iniciativas para la transición a una economía sustentable (con énfasis en la eficiencia energética), que pueden contribuir a la disminución de las emisiones de GEI por re-

\footnotetext{
${ }^{3}$ Este concepto sirve para diferir entre la gobernanza global y la que tiene lugar en el ámbito de los Estados-nación, sin intentar caer en las dificultades teóricas que impliquen el uso de otro concepto de gobernanza.
} 
gión, con beneficios globales ante las dificultades con las economías industrializadas que se han opuesto al mecanismo del Protocolo de Kioto. En sus palabras: "La RGGI y la WCI dan muestra de que la gobernanza global del cambio climático se olvidó de varios asuntos cuando diseñó instrumentos como el Protocolo de Kioto. Uno de ellos es el hecho de que la responsabilidad se debe diferenciar regionalmente. Esta condición parece necesaria para la competitividad, especialmente en un mecanismo regional como el TLCAN" (López-Vallejo, 2014: 183).

De todo lo expresado, quizás habría que cuestionar las diferencias en las posturas de los gobiernos estatales y de los locales, sobre todo si se piensa, en especial, en el caso de Estados Unidos y de Canadá, así como su posición frente al Protocolo de Kioto. La participación de sus gobiernos locales en la creación de las TGER muestra que las políticas seguidas en lo local (estados o provincias) no corresponden a las de los gobiernos estatales (representación del Estado-nación), pues se integran espacios transregionales basados en la creación de mercados de carbono. En este sentido, al margen del texto quedan algunas interrogantes, por ejemplo, ¿cuál es el papel del gobierno representativo del Estado-nación en la gobernanza global del cambio climático? y ¿por qué los gobiernos de los Estados-nación no han propuesto las TGER como alternativas para solventar los retos del cambio climático?

\section{FUENTE COMPLEMENTARIA}

Karns, Margaret P. y Karen Mingst

2004 International Organizations. The Politics and Processes of Global Governance. Boulder, Col.: Lynne Rienner.
La gobernanza global del cambio climático se olvidó de varios asuntos cuando diseñó el Protocolo de Kioto. Uno de ellos es que la responsabilidad se debe diferenciar regionalmente. 